\title{
Detection and Genetic Diversity of a Heliothine Invader (Lepidoptera: Noctuidae) From North and Northeast of Brazil
}

\author{
T. MAStrangelo,${ }^{1,2}$ D. F. PAUlo,${ }^{1}$ L. W. BERGamo,${ }^{1}$ E.G.F. MORAiS,${ }^{3}$ M. SILVA,${ }^{4}$ \\ G. BEZERRA-SILVA, ${ }^{4}$ AND A.M.L. AZEREDO-ESPIN ${ }^{1}$
}

\begin{abstract}
J. Econ. Entomol. 107(3): 000-000 (2014); DOI: http://dx.doi.org/10.1603/EC13403
ABSTRACT The cotton bollworm, Helicoverpa armigera (Hübner), was recently introduced in Brazil. During the 2012-2013 harvest, producers reported reduced yields up to 35\% on major crops. The economic losses reached US\$ 1 billion only in western Bahia, triggering a phytosanitary crisis. The deficiencies in existing taxonomic keys to deal with the morphologically indistinct larvae of $H$. armigera and the native Helicoverpa zea (Boddie) constrained the detection of new incursions of this heliothine invader. This study explored the identity of heliothine larvae that were found infesting soybean- and corn-growing areas from Roraima state, northern Brazil, through sequences of the mitochondrial cytochrome c oxidase subunit I gene. The inter- and intraspecies sequence variations of DNA barcodes in $H$. armigera and $H$. zea were analyzed. The genetic diversity and population structure of the specimens from Roraima and two populations from Piauí and Bahia states, northeastern Brazil, were assessed by adding the cytochrome c oxidase subunit II gene to the analysis. Owing to the lack of studies on genetic introgression for the two species, the suitability of using three different nuclear genes to distinguish the two species was also investigated. The results showed strong evidence that the heliothine larvae from north and northeast of Brazil are conspecific with $H$. armigera, suggesting that this invasive moth has already crossed the Amazon basin. Surveys in the north of South America should start as soon as possible to monitor the entry or spread of this moth in the Caribbean, Central America, and the United States.
\end{abstract}

KEY WORDS Helicoverpa armigera, $m t \mathrm{DNA}$, elongation factor- $1 \alpha$, isocitrate dehydrogenase, ribosomal protein S5

The cotton bollworm, Helicoverpa armigera (Hübner) (Lepidoptera: Noctuidae), is a polyphagous species, described as feeding on 181 plant species from $>60$ families (Venette et al. 2003, Pogue 2004, Srivastava et al. 2005). Each female moth can lay singly up to 1,500 eggs on several parts of the host preferably at night (European and Mediterranean Plant Protection Organization [EPPO] 1981). Larvae develop through six instars and feed on both vegetative and reproductive structures of the host (Wang and Li 1984). Large larvae (longer than $2.4 \mathrm{~cm}$ ) are the most damaging stage, as they consume $\approx 80 \%$ of their overall diet in the fifth and sixth instars (Srivastava et al. 2005). Once larvae are fully grown, they pupate in the soil and can go into diapause to overwinter (Karim 2000). H. armigera is a polyvoltine species and, depending on the weather conditions, the entire life cycle can be completed in 4-6 wk (Fitt 1989).

${ }^{1}$ Centro de Biologia Molecular e Engenharia Genética, Universidade Estadual de Campinas, Avenida Candido Rondon 400, 13083-875, Campinas, SP, Brazil.

2 Corresponding author, e-mail: mastrangelo@cbmeg.unicamp.br.

${ }^{3}$ Embrapa Roraima, BR 174, km 8, Distrito Industrial, Caixa Postal 133, 69301-970, Boa Vista, RR, Brazil.

${ }^{4}$ Universidade Estadual do Piauí (UESPI), Rua Almir Benvindo s/n, 64860-000, Uruçuí, PI, Brazil.
Owing to its dispersal capability and omnivorous behavior, $H$. armigera is the widest distributed species in the genus Helicoverpa (Venette et al. 2003) and poses a constant threat in international trade of many horticultural crops (Lammers and MacLeod 2007). Venette et al. (2003) reported 4,431 interceptions of Helicoverpa species in the United States since 1985 on fruits, vegetables, and ornamentals. From 2000-2004, Helicoverpa spp. was intercepted $>1,400$ times at United States ports, and most samples were identified to two species only, H. armigera and Helicoverpa assulta (Guenee) (Passoa 2004).

Until recently, $H$. armigera was listed as an Al quarantine pest (not present but of potential economic importance) in Brazil (Czepak et al. 2013, Embrapa 2013). However, disturbing reports started coming from a two million hectares agricultural area in the cerrado biome that encompasses parts of the Brazilian states of Maranhão, Tocantins, Piauí, and Bahia (also known as the MATOPIBA region) in early 2013. Producers, especially from western Bahia, observed population levels never seen before of larvae morphologically similar to Helicoverpa zea (Boddie), and the 2012-2013 harvest was severely affected. Infestations of larvae reduced yields up to $35 \%$ on soybean, cotton, corn, beans, sorghum, and millet. Most producers in- 
creased the number of pesticide applications by $>15 \%$, and the costs in cotton fields, for example, jumped from US\$ 400 to US $\$ 800$ per hectare. The extensive economic losses reached US $\$ 1$ billion by July 2013, generating a phytosanitary crisis at the MATOPIBA region (Embrapa 2013). Research institutes identified the new pest as $H$. armigera based mainly on the morphology of male genitalia (Hardwick 1965, Pogue 2004) and also confirmed its presence in other two states from central Brazil (Goiás and Mato Grosso; Czepak et al. 2013, Tay et al. 2013). In 1992, H. armigera provoked a similar crisis in North China, with estimated losses of US $\$ 1.3$ billion to agricultural crops in the Yellow River cotton region (Sheng 1993).

Infestations in legumes and other horticultural crops are being reported in other Brazilian states, but producers and entomologists are having trouble identifying the pest. The taxonomy based on morphological characters of Helicoverpa spp. is complicated (Pogue 2004) and presents three problems. First, as the available morphological keys are effective only for a particular life stage or gender, a large number of specimens collected from the field are discarded because they cannot be identified. Second, the use of these keys demands a high level of expertise, which increases the frequency of incorrect identifications. Finally, as $H$. armigera and $H$. zea are capable of interbreeding to produce fertile offspring (Laster and Hardee 1995), the nonmapped phenotypic variability in the characters of the hybrids could lead to misdiagnosis of the two species.

$H$. armigera and $H$. zea are difficult to distinguish especially in the larval stage. Currently, only genitalia and wing characteristics of male adults are being used to discriminate the two species (Pogue 2004, Czepak et al. 2013). The lack in Brazil of sufficient number of trained taxonomic experts in Lepidoptera and the deficiencies in existing morphology-based taxonomic keys to deal with the morphologically indistinct larvae of the two species prompted the need for a new identification approach.

In the past decade, Hebert et al. (2003) proposed the first half of the mitochondrial cytochrome c oxidase subunit I gene (COI) as the core ("DNA barcode") of a global identification system for metazoans and showed a model COI profile that was $100 \%$ successful in correctly identifying 200 specimens from closely allied species of lepidopterans. Sequences of COI and other genes from mitochondrial DNA ( $m t$ DNA) have been successfully used for population genetic studies of Lepidoptera groups (Caterino et al. 2000, Behere et al. 2007, Albernaz et al. 2012). Armstrong and Ball (2005) also demonstrated the suitability of DNA barcodes in providing a practical, cost-effective, and flexible framework for the accurate diagnostic of morphologically indistinct intercepted specimens. Increasingly, molecular diagnostic tests are accepted as an essential component of detection and identification systems of exotic invasive species (Jenkins et al. 2012).

The exact distribution and extension of economic damages of the recently introduced $H$. armigera in
Brazil is still not known. In July 2013, heliothine larvae were found infesting soybean- and corn-growing areas from Roraima state, northern Brazil. The major goal of this study was, therefore, to identify these larvae from the north by using sequences of mitochondrial genes. The interspecific and intraspecific sequence variation of the COI gene fragment ("DNA barcode") in the two morphologically alike species, $H$. armigera and $H$. zea, was also analyzed. The genetic diversity and population structure among the specimens from Roraima and two populations from Piauí and Bahia states, northeastern Brazil, were assessed by adding a second mitochondrial gene, cytochrome c oxidase subunit II gene (COII), to the analysis.

Many aspects of heliothine phylogeny remain unclear by either morphology or some molecular markers (Cho et al. 2008), and the genetic boundaries between distinct lineages of $H$. armigera and $H$. zea were not established yet owing to the lack of studies on mitochondrial introgression for the two species. In view of this, the suitability of using three different nuclear genes, which are used in phylogenomic studies with lepidopterans owing to their stability and considerable nucleotide substitution rates (Wahlberg and Wheat 2008), was investigated for the populations analyzed.

\section{Materials and Methods}

Specimens and DNA Extraction. In total, 65 heliothine larvae suspected to be $H$. armigera were collected from infested farms of eight geographic sites from the states of Roraima $(n=14)$, Piauí $(n=39)$, and Bahia $(n=12)$. The specimens from Bahia came from populations from which adult moths had been previously identified as H. armigera based on morphological characters (Czepak et al. 2013). Larvae were collected directly from host plants and immediately preserved in $100 \%$ ethanol at $-20^{\circ} \mathrm{C}$ until DNA extraction.

Total DNA was extracted from the last three abdominal segments of the larvae (Behere et al. 2013) using the phenol: chloroform method, adapted for microcentrifuge tubes (Lyra et al. 2009), resuspended in $100 \mu \mathrm{l}$ of TE buffer and stored at $-20^{\circ} \mathrm{C}$. The DNA from six $H$. zea larvae reared at laboratory for four generations (Callahan 1962) at the Piauí State University (UESPI) and two wild Spodoptera frugiperda (Smith) (Lepidoptera: Noctuidae) larvae (collected from corn in Bonfim, Roraima) was also extracted and used as control for some of the molecular analyses. Absence of cross-contamination during the extractions was confirmed by inclusion of a blank extraction among each extraction batch.

PCR Amplification and Sequencing. The partial sequences of the COI gene were amplified by PCR using the primers COIF (5'-ATTCAACCAATCATAAA GATATTGG-3') and COIR (5'-TAAACTTCTGGAT GTCCAAAAAATCA-3'; Li et al. 2011). The primers A- $t$ LEU (5'-ATGGCAGATTAGTGCAATGG-3') and B- $t$ LYS (5'-GTTTAAGAGACCAGTACTTG-3'; Liu and Beckenbach 1992) were used to amplify part of 
the COII gene. Partial sequences of three protein-encoding nuclear genes, namely elongation factor- $1 \alpha$ $(\mathrm{EF}-1 \alpha)$, isocitrate dehydrogenase (IDH), and ribosomal protein S5 (RpS5), were amplified by the pairs of primers HibEfrcm4 (5'-ATTAACCCTCACTAAAGACAGCV ACKGTYTGYCTCATRTC- $3^{\prime}$ ) and HibAlf (5'-TAAT ACGACTCACTATAGGGGAGGAAATYAARAARG AAG-3'), IDH $d e g 27 f$ (5'-TAATACGACTCACTATA GGGGGWGAYGARATGACNAGRATHATHTGG-3') and IDH $d e g \mathrm{R}$ (5'-ATTAACCCTCACTAAAGTTY TTRCAIGCCCANACRAANCCNCC-3' $),$ HibRPS5F $_{F}$ (5'-TAATACGACTCACTATAGGGATGGCNGARGA RAAYTGGAAYGA-3') and HibRPS5F $F_{R}$ (5'-ATTAA CCCTCACTAAAGCGGTTRGAYTTRGCAACACG-3';

Wahlberg and Wheat 2008), respectively.

The PCR reactions were conducted separately with $\approx 25 \mathrm{ng}$ of total DNA, $2.5 \mathrm{mM} \mathrm{MgCl} 2,0.25 \mathrm{mg} / \mathrm{ml}$ of BSA, $100 \mu \mathrm{M}$ dNTPs, $0.5 \mathrm{mM}$ of each primer, $1.5 \mathrm{U}$ of TaqDNA polymerase (Fermentas International Inc., Burlington, Canada), and $10 \times$ TaqBuffer for a final reaction volume of $25 \mu \mathrm{l}$. Amplification was carried out on a GeneAmpPCR System 9700 thermal cycler (Applied Biosystems, Foster City, CA), with the following conditions: an initial denaturation at $94^{\circ} \mathrm{C}$ for $3 \mathrm{~min}$, followed by 35 cycles of $94^{\circ} \mathrm{C}$ for $30 \mathrm{~s}, 55^{\circ} \mathrm{C}$ for $30 \mathrm{~s}\left(45^{\circ} \mathrm{C}\right.$ for COI and $48^{\circ} \mathrm{C}$ for COII $), 70^{\circ} \mathrm{C}$ for $1: 30$ $\mathrm{s}\left(72^{\circ} \mathrm{C}\right.$ for $\left.\mathrm{COII}\right)$, and ended with a $7 \mathrm{~min}$ final extension at $70^{\circ} \mathrm{C}\left(72^{\circ} \mathrm{C}\right.$ for COII $)$. After amplification, $2-\mu \mathrm{l}$ aliquots were analyzed by $1 \%$ agarose gel electrophoresis in $1 \times$ TAE $(40 \mathrm{mM}$ Tris-acetate, $1 \mathrm{mM}$ EDTA).

Amplicons were purified with the IllustraGFX kit (GE Healthcare, Bucks, UK) and sequenced bidirectionally, to ensure correct basecalling, by the ABI3730xl DNA Analyzer sequencer (Applied Biosystems, Foster City, CA), with the same primers used for the PCR reactions.

Sequence Analyses. Sequences were assembled into a contig for each specimen by using the Geneious 6.0.6 Software (Biomatters Ltd., Auckland, New Zealand), considering Phred values (Ewing et al. 1998). All sequences were aligned for each of the five genes separately using the multiple sequences alignment algorithm implemented in Clustal $\Omega$ (Sievers et al. 2011). Protein coding sequences were checked for the presence of open reading frames in MEGA 5.1 Software (Tamura et al. 2011).

Phylogenetic Analyses and Sequence Divergence. Only COI sequences were used for phylogenetic analyses owing to the lack of available sequences in public databases for the species and genetic regions investigated in this study. The 65 COI fragments from the heliothine specimens from Roraima (RR01-RR14), Piauí (PI01-PI39), and Bahia (BA01-BA12) were used to perform the phylogenetic analyses along with sequences of three S. frugiperda (two from Roraima ("S. frugiperda (01)-(02)") and a voucher under the GenBank GU090724 ("S. frugiperda (03)")), one Heliothis virescens F. (Lepidoptera: Noctuidae) (GU087832), one Helicoverpa punctigera (Wallengren) (EU768941), one Helicoverpa gelotopoeon (EU768938), one Helicoverpa hawaiiensis (EU768939), three H. assulta (GQ892856,
GQ892857, GQ892859), five H. zea ("H. zea 01-03" were from UESPI laboratory, " $\mathrm{H}$. zea 04 " was a laboratory strain from Mississippi (EU768942), and "H. zea 05" was a Brazilian voucher (HQ571107)), and the $16 \mathrm{H}$. armigera haplotypes (arm01-arm16; EU768935, EU768936, GQ892840, GQ892845, GQ892846, GQ892848, GQ892849, GQ892850, GQ892853, GQ892854, GQ995238-995244) reported by Li et al. (2011).

Behere et al. (2007) investigated the genetic diversity of $H$. armigera from Asia and Africa, but the COI sequences of this study could not be compared with theirs because they used only a $511 \mathrm{bp}$ fragment located in the region $980-1490$ of the COI gene that was not overlapped with the region 39-696 analyzed in this study and by Li et al. (2011).

Neighbor-joining (NJ) distance analysis (Saitou and Nei 1987) and sequence divergences were calculated in MEGA 5.1 software (Tamura et al. 2011) with Kimura two-parameters (K2P; Kimura 1980) and uncorrected sequence divergences ( $p$-distances) models. Node supports were measured by 5,000 replicates of bootstrap (BS).

The Bayesian inference (BI) and maximum-likelihood (ML) methods were also used for the phylogenetic analyses. The best fitted substitution model selection for the dataset was carried out using MrAIC 1.4.4 (Nylander 2004) software. The favored model for COI was the GTR+I (General Time Reversible; I = invariable sites). BI analysis was conducted using $\mathrm{mr}$ Bayes v3.1.2 (Ronquist and Huelsenbeck 2003) with the favored substitution model selected previously. Two independent analyses were run for $10,000,000$ generations (sample frequency $=1,000$ ), with $25 \%$ of burn-in after checking for convergence. Node supports were analyzed by their posterior probabilities in the $70 \%$ majority rule consensus tree. The ML analysis was conducted using PhyML (Guindon and Gascuel 2003) with SPR tree topology search operation, 10 random starting trees as parameters and the MrAIC 1.4.4-favored substitution model. Node supports were accessed with 1,000 replicates of BS.

The DnaSP.V5 software (Librado and Rozas 2009) was used to investigate polymorphisms in COI sequences and to identify possible diagnostic characters for species discrimination between H. armigera and $H$. zea.

A haplotype network was constructed based on the 96 COI sequences used in the phylogenetic analyses by TCS version 1.21 (Clement et al. 2000), following the guidelines proposed by Bandelt et al. (2000). The connection between haplotypes was limited by a probability of parsimony for DNA pairwise differences lower than $95 \%$.

Structure and Genetic Diversity of the Populations Sampled. The partial sequences of the COI and COII genes from the 65 heliothine specimens from Roraima, Piauí, and Bahia were concatenated for the genetic diversity analyses. Individual sequences were collapsed in haplotypes and, in the absence of detailed knowledge of gene flow and for the purpose of this study, each one of the three Brazilian states sampled was regarded as a different "population" in the anal- 
yses. Haplotype frequencies, haplotype diversity $(\hat{\mathbf{H}})$, and nucleotide diversity $(\pi)$, as defined by Nei (1987), were estimated using Arlequin v.3.5 (Excoffier and Lischer 2010).

Analysis of molecular variance (AMOVA) was performed to access the genetic structure among and within the three populations as implemented in Arlequin v.3.5 (Excoffier and Lischer 2010). Genetic differences among populations were determined through pairwise $\mathbf{F}_{\mathrm{ST}}$ statistics (Reynolds et al. 1983, Slatkin 1995). Statistical significance was accessed by 10,000 permutations, the computed distance matrix used pairwise difference, and the gamma $\alpha$ value was considered zero for the analysis.

Sequence Divergence Using Nuclear Genes. The partial sequences of the nuclear genes EF- $1 \alpha$, IDH, and RpS5 for $6 \mathrm{H}$. zea from laboratory rearing and 22 heliothine larvae from Roraima, Piauí, and Bahia (one representative from each one of the 22 different haplotypes resulting from the concatenation of the COI and COII fragments) were used for the estimation of inter- and intraspecific genetic distances between $H$. $z e a$ and $H$. armigera. Sequence divergence with K2P (Kimura 1980) and uncorrected sequence divergence ( $p$-distances) models were calculated by using MEGA 5.1 software (Tamura et al. 2011).

\section{Results}

All specimens yielded high-quality DNA and were successfully sequenced for the fragments of COI (658 bp), COII (554 bp), EF-1 $\alpha$ (779 bp), IDH (560 bp), and RpS5 (593 bp) genes. The COI sequences obtained for the specimens from Roraima, Piauí, and Bahia were blasted against GenBank database and checked in BOLD Identification System (BOLD 2007), and all the 65 heliothine larvae matched 99$100 \%$ with $H$. armigera sequences. Chromatograms were reliable for all the gene fragments and, after terminal cutoff owing to sequences ambiguities, the absence of indels made the alignment straightforward. The $m t$ DNA and nuclear sequences generated in this study have been deposited in GenBank (KF624811KF625029).

Phylogenetic Analyses and Sequence Divergence. Four different phylogenetic analyses (NJ under $p$-distance and K2P models, BI, and ML under MrAIC 1.4.4-favored substitution model) conducted for the COI sequences gave nearly identical tree topologies (Fig. 1). NJ and BI proved to be reliable measures of species delimitation, as all identifiable species with more than one sequence (S. frugiperda, H. assulta, and H. zea) had all individuals joined by $>98 \%$ BS and 1.0 posterior probability (PP). The 65 heliothine specimens from Roraima, Piauí, and Bahia joined by $>95 \%$ BS and 0.9 PP values formed a monophyletic group with the $16 \mathrm{H}$. armigera sequences reported by $\mathrm{Li}$ et al. (2011). The H. armigera group formed a robustly supported monophyletic clade with $H$. zea $(80 \% / 77 \%$ NJ BS, $0.91 \mathrm{BI}$ PP, and 79\% ML BS), as expected for these species (Cho et al. 2008).
The genetic distance (Table 1) between the investigated heliothine specimens from Roraima, Piauí, and Bahia and the $16 \mathrm{H}$. armigera $\mathrm{COI}$ sequences reported by $\mathrm{Li}$ et al. (2011) revealed very low sequence divergence $(0-0.8 \%)$, suggesting conspecificity among the specimens (Avise 2000). For instance, the sequence divergence between the 65 heliothine specimens and the $H$. zea group exceed that divergence, ranging approximately from 2 to $3 \%$, likely the range observed between the $H$. armigera group and H.zea (Table 1). For both distance models used (K2P and $p$-distance), there was substantial sequence divergence between the 65 heliothine specimens and the four other species of the genus Helicoverpa $(\approx 2.6-6.3 \%)$, He. virescens $(\approx 7-$ $8 \%$ ), and S. frugiperda ( $\approx 10-12 \%$; Table 1$)$.

The sequences from the 65 heliothine specimens (RR01-RR14; PI01-PI39; and BA01-BA12) and the 16 H. armigera (arm1-arm16; $\mathrm{Li}$ et al. 2011) were collapsed into 21 different haplotypes, which were linked in a unique parsimony network (Fig. 2). The general topology of the network showed one clade containing all $H$. armigera haplotypes, separated by $>13$ mutational steps from $H$. zea and 11 steps from the other heliothine species. The two most common H. armigera COI haplotypes, designated by $\mathrm{Li}$ et al. (2011) as "arml" (found in Thailand and the Chinese province of Yunnan) and "arm2" (found in Yunnan and Henan provinces, China), are most likely to be ancestral (Castelloe and Templeton 1994). Five specimens from Roraima, 18 from Piauí, and 8 from Bahia were identical to "arm1," and 6, 11, and 2 specimens from Roraima, Piauí, and Bahia, respectively, were identical to "arm2" (Fig. 2). Most of the other H. armigera haplotypes were rare (single individuals) and their frequency was comparatively high (15 out of 21 ). Haplotypes of $H$. armigera differed from the center of the network by no more than three mutational steps. No haplotype was shared among the six Helicoverpa species, and He. virescens differed by 62 mutational steps from the H. armigera group (Fig. 2).

The DNA barcodes generated for $H$. armigera and H. zea (Table 2) based on combinations of diagnostic nucleotides allowed the observation of 30 nucleotide substitutions between the two species, with 14 unique diagnostic characters that could be used to discriminate the species.

Structure and Genetic Diversity of the Populations Sampled. The COI and COII fragments from the 65 specimens from Roraima, Piauí, and Bahia were concatenated, generating a final 1212-bp sequence for each individual. In total, 22 different haplotypes were defined (Fig. 3). Haplotypes were shared among samples from the three Brazilian states, especially the haplotype one, the most frequent, suggesting a significant gene flow among these regions. In general, high haplotype diversity ( $\mathrm{H}$-mean $>0.85)$ and low nucleotide diversity $(\pi$-mean $<0.003)$ were observed for the locations analyzed (Fig. 3). AMOVA detected no genetic structure $\left(\mathrm{F}_{\mathrm{ST}}=7.10^{-5} ; P=0.44\right)$, with $99.9 \%$ of variation accounted for at the within population level and with only $0.01 \%$ variation observed among 


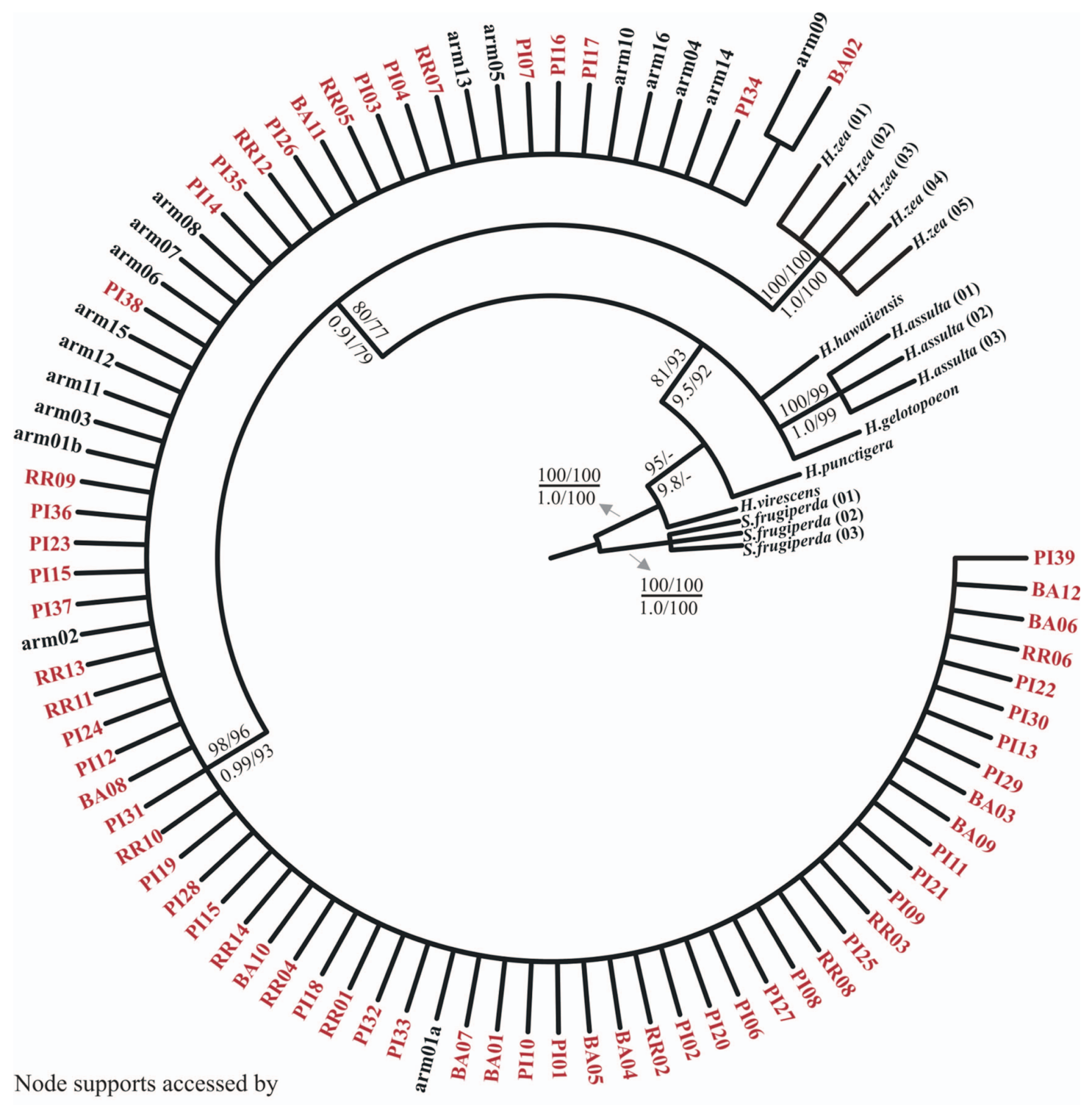

Bootstrap $p$-distance under NJ / K2P under NJ

Posterior Probability under BI / Bootstrap under ML

Fig. 1. NJ strict consensus tree (topology under $p$-distance model) inferred using COI complete dataset for Heliothine species. The 65 specimens from Roraima (RR01-RR14), Piauí (PI01-PI39), and Bahia (BA01-BA12) are in red. Sequences of S. frugiperda, He virescens, and H. punctigera were used as outgroups. Numbers above branches refer to NJ BS proportions among 5,000 replicates, while numbers below branches refers to BI PP and ML BS proportions among 1,000 replicates. The four analyses gave nearly identical tree topologies, and node supports values below $70 \%$ (BS), 0.7 (PP), or both, were not recorded in the tree $(-)$.

populations. Pairwise $\mathrm{F}_{\mathrm{ST}}$ values (considering the individual three Brazilian states as separate populations) were low and not significant $\left(\mathrm{F}_{\mathrm{ST}}<0.03 ; P>0.05\right)$ in the studied specimens (Table 3 ).

Sequence Divergence Using Nuclear Genes. The genetic distances between 22 heliothine specimens from Roraima, Piauí, and Bahia and $6 \mathrm{H}$. zea from laboratory rearing estimated by three different nu- clear genes were low (K2P and uncorrected $p$-distance $<3 \%$; Table 4 ). The highest number of variable sites $(2.7 \%)$ was observed for the fragments of the RpS5 gene, but the highest sequence divergence $(2.2 \%)$ was given by the EF- $1 \alpha$ gene. As the intraspecific range of differences from both $H$. armigera and $H$. zea overlapped the interspecific ranges (Table 4), the three nuclear gene fragments investigated failed to 
Table 1. Mitochondrial DNA COI pairwise genetic distances for the investigated heliothine specimens from Brazil and other Lepidoptera species

\begin{tabular}{|c|c|c|c|c|}
\hline \multicolumn{3}{|c|}{ Species compared } & \multirow{2}{*}{$\frac{\text { Kimura two-parameters (K2P) }}{\text { Range }}$} & \multirow{2}{*}{$\begin{array}{c}\begin{array}{c}\text { Uncorrected sequence } \\
\text { divergences }(p \text {-distance })\end{array} \\
\text { Range }\end{array}$} \\
\hline & & & & \\
\hline \multirow[t]{8}{*}{ Investigated Heliothine specimens ${ }^{a}$} & vs & S. frugiperda & $0.114-0.120$ & $0.099-0.103$ \\
\hline & & He. virescens & $0.076-0.079$ & $0.068-0.071$ \\
\hline & & H. punctigera & $0.058-0.063$ & $0.053-0.058$ \\
\hline & & H. gelotopoeon & $0.035-0.040$ & $0.033-0.038$ \\
\hline & & H. assulta & $0.032-0.37$ & $0.030-0.035$ \\
\hline & & H. hawaiiensis & $0.027-0.032$ & $0.026-0.030$ \\
\hline & & H. zea & $0.023-0.027$ & $0.024-0.029$ \\
\hline & & H. armigera & $0.000-0.008$ & $0.000-0.008$ \\
\hline H. armigera $^{b}$ & vs & H. zea & $0.026-0.031$ & $0.024-0.029$ \\
\hline
\end{tabular}

${ }^{a}$ The investigated heliothine specimens comprised the 65 larvae sampled from Roraima, Piauí, and Bahia states.

${ }^{b}$ The $H$. armigera group encompassed only the 16 COI haplotypes described by Li et al. (2011).

robustly discriminate the two morphologically alike species.

\section{Discussion}

The $m t$ DNA COI phylogeny strongly supported that the 65 heliothine larvae from Roraima, Piauí, and Bahia are conspecific with $H$. armigera. The four phylogenetic analyses grouped these specimens with $16 \mathrm{H}$. armigera COI sequences described by Li et al. (2011) as a single monophyletic group. The other defined species (S. frugiperda, He. virescens, and the other five Helicoverpa species) were recovered in distinct positions or clades on strongly supported branches (Fig. $1)$. The $H$. zea clade was monophyletic with the $H$. armigera clade, and this same phylogenetic pattern had been observed in previous studies (Fang et al. 1997, Cho et al. 2008). Behere et al. (2007) suggested that the American H. zea populations were established via founder event from $H$. armigera (or from their joint common ancestor; Mallet et al. 1993) no >1.5 million years ago, which is further supported by the high similar morphology and the possibility of mating compatibility between the two species (Laster et al. 1985, Laster and Hardee 1995, Laster and Sheng 1995).

Despite the recent history of separation between the two species (Behere et al. 2007) and the low sequence divergences expected for the order Lepidoptera (Caterino et al. 2000, Hebert et al. 2003), the information content of the COI fragment selected in this study was sufficient to enable recognition of the divergence among the lepidopterans analyzed. The estimated genetic distances (K2P and $p$-distance) indicated divergence values between species $>2 \%$. Setting this value as a threshold for species diagnosis in this study allowed the discrimination of all heliothine species analyzed (Table 1$)$. The low divergence $(0-$ $0.8 \%$ ) between the 65 heliothine specimens from north and northeast of Brazil and the $H$. armigera sequences from Li et al. (2011) indicated conspecificity among these individuals, and both groups diverged similarly with $H$. zea $(\approx 2-3 \%$ for $\mathrm{K} 2 \mathrm{P}$ and $p$-distance;Table 1). Hebert et al. (2003) also designated a 2-3\% $m t$ DNA COI sequence divergence as a "threshold" to discriminate insect and mammal spe- cies. However, as rates of genetic change between taxa are dynamic processes (Rubinoff et al. 2006), thresholds used to define species may vary widely among studies with lepidopterans. Hebert et al. (2004) used a threshold as low as $0.32 \%$ for skipper butterflies, and Whinnett et al. (2005) observed divergences from 0.23 to $6.4 \%$ in Ithoninae butterflies, demonstrating that species thresholds can be extremely fluid.

The $m t$ DNA COI haplotype network showed that the 65 heliothine samples from Roraima, Piauí, and Bahia formed a homogeneous group of $\mathrm{H}$. armigera haplotypes (Fig. 2). The connections between haplotypes was similar to that reported by Li et al. (2011), with $H$. armigera haplotypes differing from the center of the network by no more than three mutational steps and no shared haplotypes among the different species. Two Chinese haplotypes, "arml" (EU768935) and "arm2" (EU768936; Cho et al. 2008, Li et al. 2011), encompassed 47.7 and $29.2 \%$, respectively, of the haplotypes found in the samples from Roraima, Piauí, and Bahia, indicating that "arml" is a potential ancestral haplotype of H. armigera. H. zea appeared linked to the $H$. armigera group (by 15 mutational steps) through the haplotypes from samples PI-34, PI-3, PI-4, and RR-7 (Fig. 2). In the haplotype network reported by Behere et al. (2007), the H. armigera and H. zea groups were separated by $\approx 20$ mutational steps.

This study observed shared haplotypes between the three Brazilian states, not significant $\mathrm{F}_{\mathrm{ST}}$ values, and low nucleotide diversity between the sampled regions from north and northeast of Brazil (Table 3; Fig. 3). In general, high values of haplotype diversity and low nucleotide diversity, combined with a high number (15 out of 22) of low frequency haplotypes (Fig. 3), are characteristic of species that have undergone a process of recent population expansion (Excoffier et al. 2009).

Many authors, using different molecular methods, have verified similar patterns of genetic variation, which seems to be common in insect pests capable of rapid spatial expansion and long-range dispersion such as H. armigera. Daly and Gegg (1985) observed very little genetic variation $\left(\mathrm{F}_{\mathrm{ST}}=0.012\right)$ between Australian $H$. armigera populations from a $3,000-\mathrm{km}$ study area using isozymes. Nibouche et al. (1998) verified 


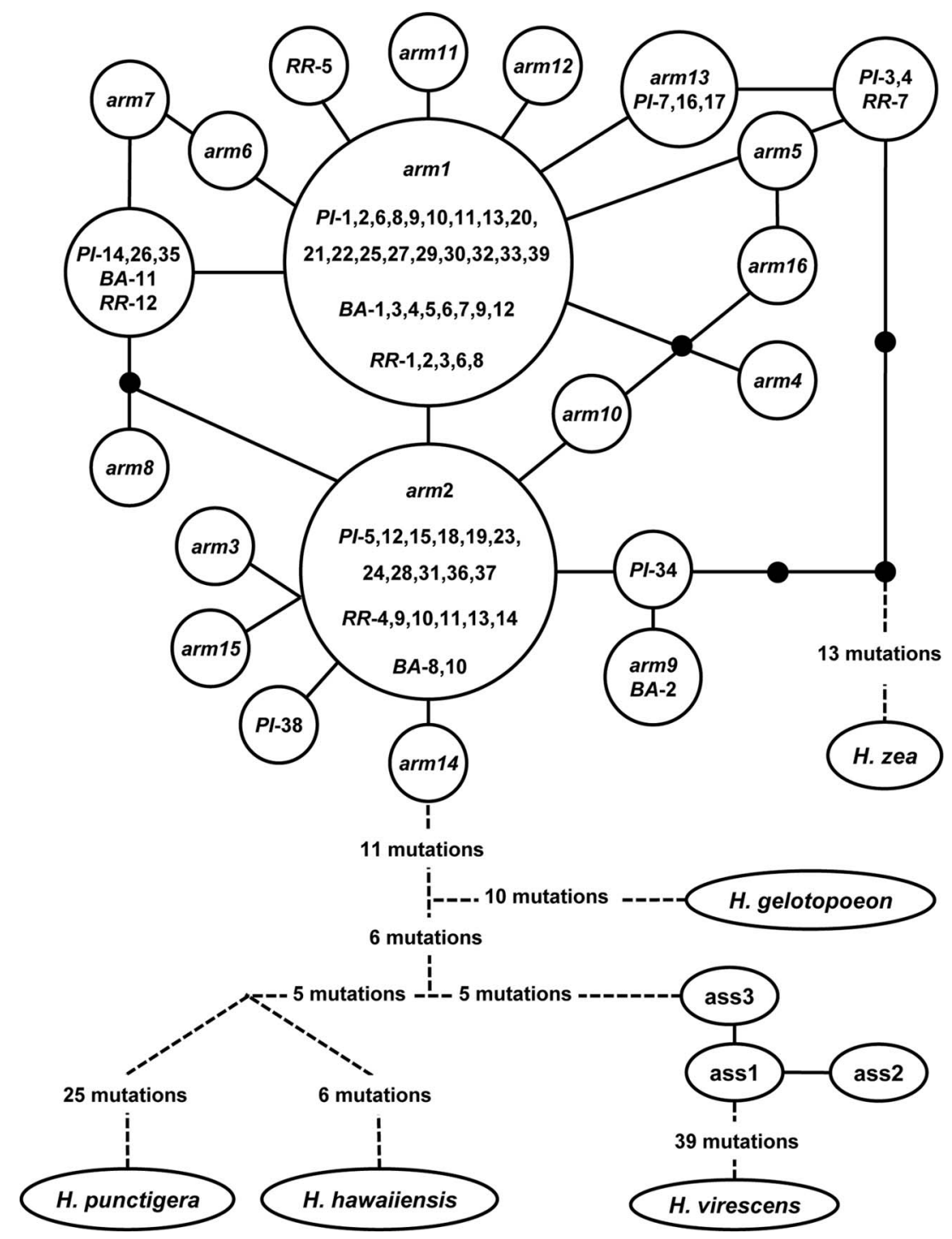

Fig. 2. Haplotype network based on partial $m t$ DNA COI (658 bp) of heliothine species. Each haplotype is represented by a circle. The original names of the haplotypes reported by Li et al. (2011) (arm1-arm16) and the 65 specimens from Roraima (RR01-RR14), Piauí (PI01-PI39), and Bahia (BA01-BA12) were maintained. Full lines between haplotypes represent one mutational step, while the dots are presumptive intermediate haplotypes that were not observed.

that barriers such as the Sahara desert had not prevented long-distance migration in $H$. armigera, as no significant isozyme allele frequency differences were found between populations from either side of the desert. Zhou et al. (2000) reported very low genetic distances among Israeli and Turkish H. armigera populations using RAPD-PCR analysis. Using microsatellite loci, Endersby et al. (2007) found no evidence of genetic structure among samples from Australia and New Zealand collected at different times. Behere et al. (2007) suggested the occurrence of long-distance gene flow in $H$. armigera, based on low $\mathrm{F}_{\mathrm{ST}}$ values and low among-group haplotype variance across Australia, China, India, Pakistan, Burkina Faso, and Uganda. No significant population substructure across India, irre- spective of cropping seasons, were inferred from EPIC-PCR DNA markers (Behere et al. 2013).

Long-distance migration of this heliothine pest has also been suggested by capture of adult moths on Ascension Island $(2,000 \mathrm{~km}$ from the African coast; Widmer and Schofield 1983) and Willis Island in the Coral Sea (450 km from the coast of Australia; Daly and Gregg 1985). Borne by wind, H. armigera can reach the United Kingdom from southern Europe (>1,000 km; Pedgley 1985). Mark-recapture experiments have shown that $H$. armigera moths could fly $200-300 \mathrm{~km}$ in a single night (Armes and Cooter 1991).

Correct and prompt detection of this highly mobile invasive pest is an essential step before initiating quarantine actions, proper control measures, and quick 
Table 2. DNA barcodes for $H$. armigera and $H$. zea based on combinations of diagnostic nucleotides represented by the International Union of Pure and Applied Chemistry symbols

\begin{tabular}{rccccc}
\hline \hline N.P $^{a}$ & H. armigera & H. zea & N.P & H. armigera & H. zea \\
\hline 40 & R & A & 340 & C & T \\
85 & Y & T & 346 & M & A \\
115 & $\boldsymbol{T}^{b}$ & C & 368 & R & G \\
127 & $\boldsymbol{T}$ & C & 385 & Y & T \\
130 & R & A & 386 & C & T \\
181 & K & T & 418 & C & T \\
217 & A & G & 439 & Y & T \\
238 & C & T & 477 & G & A \\
262 & R & A & 478 & $C$ & T \\
268 & $\boldsymbol{T}$ & C & 496 & R & A \\
274 & Y & T & 536 & $\boldsymbol{T}$ & C \\
277 & $\boldsymbol{T}$ & C & 539 & Y & T \\
278 & $\boldsymbol{T}$ & C & 548 & $\boldsymbol{T}$ & C \\
286 & R & A & 595 & Y & C \\
313 & Y & T & 616 & Y & T \\
\hline
\end{tabular}

${ }^{a}$ N.P. indicates the nucleotide position which is relative to the beginning of the fragment investigated in this study.

${ }^{b}$ The 14 pure diagnostic characters are shaded in bold italics.

response to any incursion ( $\mathrm{Li}$ et al. 2011). The current study demonstrated the effectiveness of DNA barcodes for distinguishing $H$. armigera from $H$. zea (Table 2). The use of DNA barcodes has also succeeded in species-level identification for $>50,000$ species of Lepidoptera so far (Hebert et al. 2004, Hajibabaei et al. 2006, Burns et al. 2008, Silva-Brandão et al. 2009, Jinbo et al. 2011).

Despite the high resolution to distinguish the six Helicoverpa species analyzed, DNA barcodes should not be used blindly in the context of biosecurity (Arm-
Table 3. Pairwise $\mathbf{F}_{\mathrm{ST}}$ estimates among three investigated heliothine populations from Brazil based on combined genes COI and COII

\begin{tabular}{lccc}
\hline \hline Populations & Piauí & Bahia & Roraima \\
\hline Piauí & 0.0000 & & \\
Bahia & $0.0255(P=0.15)$ & 0.0000 & \\
Roraima & $-0.0056(P=0.48)$ & $-0.041(P=0.91)$ & 0.0000 \\
\hline
\end{tabular}

strong and Ball 2005, Darling and Blum 2007). There are some shortcomings of $m t$ DNA for "barcoding" identification, which includes recombination, inconsistent mutation rate, heteroplasmy, maternal inheritance, and introgression (Rubinoff et al. 2006, Krishnamurthy and Francis 2012). Although the possibility of mating incompatibility via mechanical isolation between $H$. armigera and $H$. zea was reported (Hardwick 1965), mating compatibility between laboratory $H$. zea from the United States and wild $H$. armigera from Asia and Australia under controlled conditions (Laster et al. 1985, Laster and Hardee 1995, Laster and Sheng 1995 ) is also possible. These studies, allied to the inexistence of hybridization trials between $H$. armigera and H. zea populations from Central and South America, raise the question if the 65 heliothine larvae from north and northeast of Brazil are from a pure strain of the recently introduced $H$. armigera or a hybrid originated from the cross between a $H$. zea male and a $H$. armigera female (Caterino et al. 2000, Hebert et al. 2003).

New gene regions have been successfully sequenced for Lepidoptera and primers for the genes

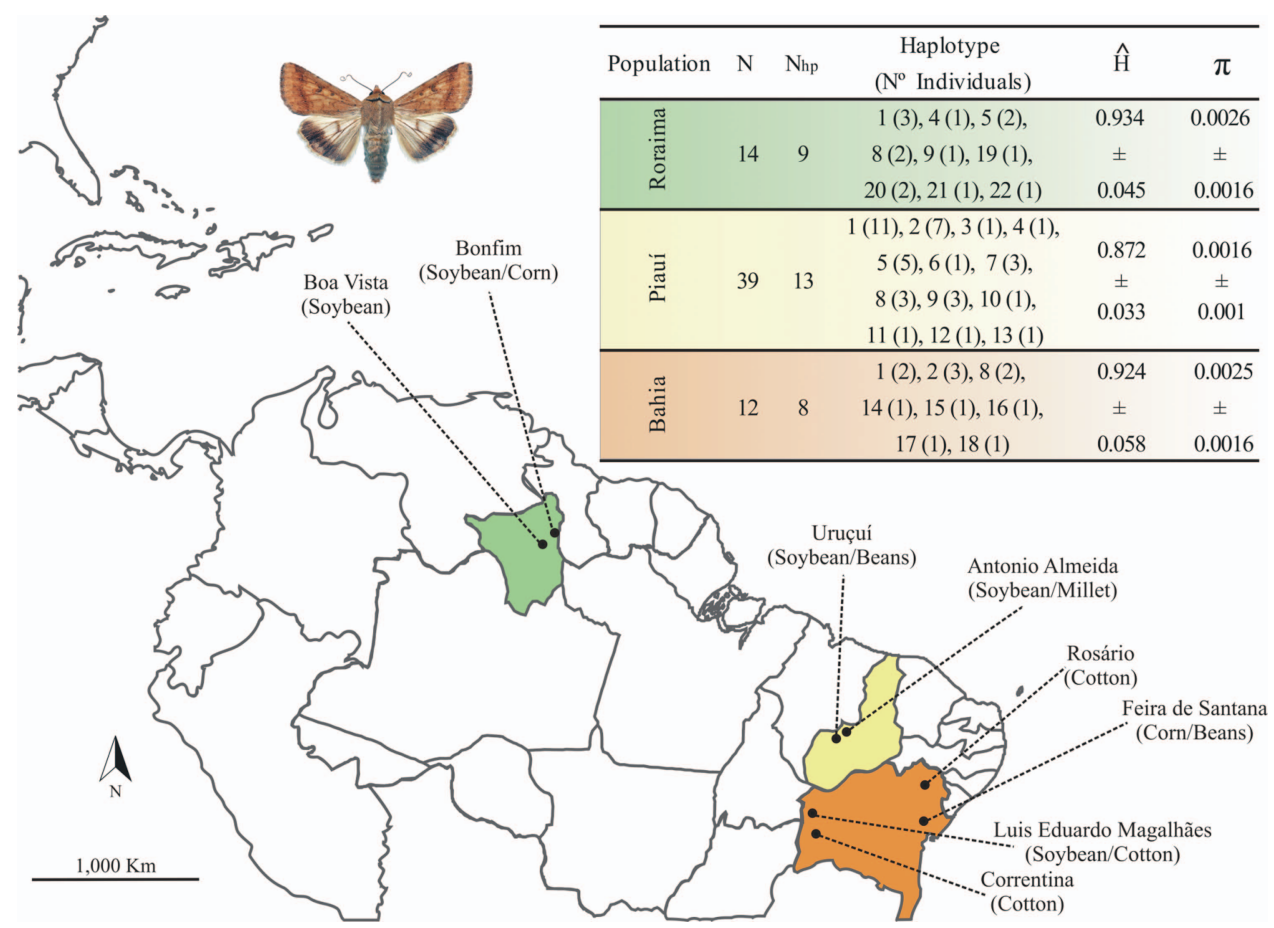

Fig. 3. Heliothine sampled sites, haplotype distribution, and genetic variability indices $( \pm \mathrm{SE})$. 
Table 4. Interspecies and intraspecies divergences given by three nuclear genes for the $H$. armigera and $H$. $z e a$ investigated

\begin{tabular}{|c|c|c|c|c|c|c|c|c|c|}
\hline \multirow{3}{*}{ Gene } & \multicolumn{9}{|c|}{ Genetic distances } \\
\hline & \multirow{2}{*}{ No. of specimens sequenced } & \multirow{2}{*}{$\begin{array}{l}\text { Amplicon } \\
\text { length }\end{array}$} & \multirow{2}{*}{$\begin{array}{c}\text { No. of } \\
\text { variable sites }\end{array}$} & \multicolumn{2}{|c|}{ Interspecific } & \multicolumn{2}{|c|}{ Intra. H.armigera } & \multicolumn{2}{|c|}{ Intra. H. zea } \\
\hline & & & & $\mathrm{K} 2 \mathrm{P}$ & $p$-distance & $\mathrm{K} 2 \mathrm{P}$ & $p$-distance & $\mathrm{K} 2 \mathrm{P}$ & $p$-distance \\
\hline $\mathrm{EF}-1 \alpha$ & $28(6 \mathrm{H}$. zea and $22 \mathrm{H}$. armigera & $779 \mathrm{bp}$ & $17(2.1 \%)$ & $0.004-0.022$ & $0.004-0.022$ & $0-0.008$ & $0-0.006$ & $0-0.022$ & $0-0.022$ \\
\hline IDH & & $560 \mathrm{bp}$ & $7(1.25 \%)$ & $0.004-0.018$ & $0.004-0.018$ & $0-0.008$ & $0-0.008$ & 0 & 0 \\
\hline RpS5 & & $593 \mathrm{bp}$ & $16(2.7 \%)$ & 0-0.019 & $0-0.018$ & $0-0.017$ & $0-0.016$ & 0 & 0 \\
\hline
\end{tabular}

$\mathrm{EF}-1 \alpha$, IDH, and RpS5 were suggested to be universal in Lepidoptera, giving robustness for most species (Wahlberg and Wheat 2008). Trying to overcome the unresolved hybridization issue, sequence divergence between $H$. zea and H. armigera was also explored using these three nuclear genes. Nonetheless, the variation of the fragments analyzed was not sufficient to robustly discriminate $H$. armigera from $H$. zea (Table 4 ). The highest sequence divergence obtained was $2.2 \%$, but inter- and intraspecific divergences were overlapped. In general, nuclear DNA undergoes relatively slow mutation compared with $m t \mathrm{DNA}$, which may provide for the first a smaller degree of taxonomic resolution at the species level (Waugh 2007). A monophyletic relationship between $H$. zea and $H$. armigera was inferred previously based on the EF- $1 \alpha$ gene (Cho et al. 1995) and the Dopa Decarboxylase (DDC) gene (Fang et al. 1997). The insufficient variation in the $\mathrm{EF}-1 \alpha, \mathrm{IDH}$, and RpS5 genes to accurately reconstruct the recent divergence between the species may be a consequence of the common ancestry (Mallet et al. 1993, Behere et al. 2007).

Further studies to search for combining evidence from $m t$ DNA and more rapidly evolving nuclear DNA markers with pure strains and hybrids from $H$. armigera and $H$. zea will be necessary to enhance the robustness of any key and assignment of identification for these two species. While comprehensive protocols with new molecular markers giving higher taxonomic resolution are not developed, $m t \mathrm{DNA}$-based identification systems will undoubtedly continue to provide diagnostic and geographic origin information that exceeds that which can be achieved by using morphological studies alone (Armstrong and Ball 2005, Behere et al. 2007). The $m t$ DNA-based systems can also help to speed the work of policy makers interested in the anticipatory detection of heliothine invasive species.

The strong evidence that the heliothine larvae from Roraima are conspecific with $H$. armigera under significant gene flow with northeastern populations suggest that this invasive moth is not restricted to crop fields from northeast and central Brazil and probably already has crossed the Amazon basin. The data of the current study, however, do not allow to precise the likely source of the invasions. Tay et al. (2013) were also unable to define the origin of the $H$. armigera introduced in Brazil, but suggested that the samples from Mato Grosso might have come from either multiple recent incursions or a single incursion from mixed populations of $H$. armigera.

A broader geographic sampling in northern Brazil and surveys in Venezuela and Colombia should start as soon as possible to monitor its entry or spread in the Caribbean, Central America, and the United States. The increasing rates of $H$. armigera infestations in Brazil were favored by many conditions (tropical climate, extensive agriculture, wide host availability, insecticide resistance, and drought in northeastern states; Behere et al. 2013, Czepak et al. 2013). Finding similar conditions in other regions of America, this heliothine invader could trigger more phytosanitary crisis.

\section{Acknowledgments}

We thank Francisco dos Santos Silva from UESPI and Suely Brito from the Agência de Defesa Agropecuária da Bahia (ADAB) for some heliothine samples from Piauí and Bahia states, respectively. We are thankful to Rosangela A. Rodrigues for technical and laboratory assistance, and to the Fundação de Amparo à Pesquisa do Estado de São Paulo (FAPESP) for a fellowship.

\section{References Cited}

Albernaz, K. C., K. L. Silva-Brandao, P. Fresia, F. L. Consoli, and C. Omoto. 2012. Genetic variability and demographic history of Heliothis virescens (Lepidoptera: Noctuidae) populations from Brazil inferred by $m t$ DNA sequences. Bull. Entomol. Res. 102: 333-343.

Armstrong, K. F., and S. L. Ball. 2005. DNA barcodes for biosecurity: invasive species identification. Phil. Trans. R. Soc. B 360: 1813-1823.

Armes, N. J., and R. J. Cooter. 1991. Effects of age and mated status on flight potential of Helicoverpa armigera (Lepidoptera: Noctuidae). Physiol. Entomol. 16: 131-144.

Avise, J. C. 2000. Phylogeography: the history and formation of species. Harvard University Press, Cambridge, MA.

Bandelt, H. J., V. Macaulay, and M. Richards. 2000. Median networks: speedy construction and greedy reduction, one simulation, and two case studies from human $m t$ DNA. Mol. Phylogenet. Evol. 16: 8-28.

Behere, G. T., W. T. Tay, D. A. Russell, D. G. Heckel, B. R. Appleton, K. R. Kranthi, and P. Batterham. 2007. Mitochondrial DNA analysis of field populations of Helicoverpa armigera (Lepidoptera: Noctuidae) and of its relationship to H. zea. BMC Evol. Biol. 7: 117-127.

Behere, G. T., W. T. Tay, D. A. Russell, K. R. Kranthi, and P. Batterham. 2013. Population genetic structure of the cotton bollworm Helicoverpa armigera (Hubner) (Lepidoptera: Noctuidae) in India as Inferred from EPIC-PCR DNA Markers. PLoS ONE 8: e53448.

(BOLD) The Barcode of Life Data System. 2007. Bold.v3. Mol. Ecol. Notes 7: 355-364. (http://www.boldsystems. org/index.php/IDS_OpenIdEngine).

Burns, J. M., D. H. Janzen, M. Hajibabaei, W. Hallwachs, and P.D.N. Hebert. 2008. DNA barcodes and cryptic species 
of skipper butterflies in the genus Perichares in Area de Conservacion Guanacaste, Costa Rica. Proc. Natl. Acad. Sci. U.S.A. 105: 6350-6355.

Callahan, P. S. 1962. Techniques for rearing the corn earworm, Heliothis zea. J. Econ. Entomol. 55: 453-457.

Castelloe, J., and A. R. Templeton. 1994. Root probabilities for intraspecific gene trees under neutral coalescent theory. Mol. Phylogenet. Evol. 3: 102-113.

Caterino, M. S., S. Cho, and F. A. Sperling. 2000. The current state of insect molecular systematics: a thriving Tower of Babel. Annu. Rev. Entomol. 45: 1-54.

Cho, S., A. Mitchell, J. C. Regier, C. Mitter, R. W. Poole, T. P. Friedlander, and S. Zhao. 1995. A highly conserved nuclear gene for low-level phylogenetics: Elongation factor$1 \alpha$ recovers morphology-based tree for Heliothine moths. Mol. Biol. Evol. 12: 650-656.

Cho, S., A. Mitchell, C. Mitter, J. Regier, M. Matthews, and R. Robertson. 2008. Molecular phylogenetics of heliothine moths (Lepidoptera: Noctuidae: Heliothinae), with comments on the evolution of host range and pest status. Syst. Entomol. 33: 581-594.

Clement, M., D. Posada, and K. A. Crandall. 2000. TCS: a computer program to estimate gene genealogies. Mol. Ecol. 9: 1657-1659.

Czepak, C., K. C. Albernaz, L. M. Vivan, H. O. Guimarães, and T. Carvalhais. 2013. First reported occurrence of Helicoverpa armigera (Hübner) (Lepidoptera: Noctuidae) in Brazil. Pesqui. Agropec. Trop. 43: 110-113.

Daly, J. C., and P. Gregg. 1985. Genetic variation in Heliothis in Australia: species identification and gene flow in two pest species $H$. armigera (Hübner) and $H$. punctigera Wallengren (Lepidoptera: Noctuidae). Bull. Entomol. Res. 75: 169-184.

Darling, J. A., and M. J. Blum. 2007. DNA-based methods for monitoring invasive species: a review and prospectus. Biol. Invasions 9: 751-765.

(Embrapa) Empresa Brasileira de Pesquisa Agropecuária. 2013. Ações emergenciais propostas pela Embrapa para o manejo integrado de Helicoverpa spp. para áreas agrícolas, 2013. (http://www.cpac.embrapa.br/publico/usuarios/ uploads/destaques/manejo_helicoverpa2.pdf).

Endersby, N. M., A. A. Hoffmann, S. W. McKechnie, and A. R. Weeks. 2007. Is there genetic structure in populations of Helicoverpa armigera from Australia? Entomol. Exp. Appl. 122: 253-263.

(EPPO) European and Mediterranean Plant Protection Organization. 1981. Data sheets on quarantine organisms $n^{\circ}$ 110: Helicoverpa armigera. Paris: EPPO. (Bulletin, 11).

Excoffier, L., and H.E.L. Lischer. 2010. Arlequin suite ver 3.5: A new series of programs to perform population genetics analyses under Linux and Windows. Mol. Ecol. Res. 10: 564-567.

Excoffier, L., M. Foll, and R. J. Petit. 2009. Genetic consequences of range expansions. Annu. Rev. Ecol. Evol. Syst. 40: 481-501.

Ewing, B., L. Hillier, M. Wendl, and P. Green. 1998. Base calling of automated sequencer traces using phred. I. Accuracy assessment. Genome Res. 8: 175-185.

Fang, Q. Q., S. Cho, J. C. Regier, C. Mitter, M. Matthews, R. W. Poole, T. P. Friedlander, and S. Zhao. 1997. A new nuclear gene for insects phylogenetics: Dopa Decarboxylase is informative of relationships within Heliothinae (Lepidoptera: Noctuidae). Syst. Biol. 46: 269-283.

Fitt, G. P. 1989. The ecology of Heliothis species in relation to agroecosystems. Annu. Rev. Entomol. 34: 17-52.

Guindon, S., and O. Gascuel. 2003. A simple, fast and accurate algorithm to estimate large phylogenies by maximum likelihood. Syst. Biol. 52: 696-704.
Hajibabaei, M., D. H. Janzen, J. M. Burns, W. Hallwachs, and P.D.N. Hebert. 2006. DNA barcodes distinguish species of tropical Lepidoptera. Proc. Natl. Acad. Sci. U.S.A. 103: 968-971.

Hardwick, D. F. 1965. The corn earworm complex. Mem. Entomol. Soc. Can. 40: 1-247.

Hebert, P.D.N., A. Cywinska, S. L. Ball, and J. R. deWaard. 2003. Biological identifications through DNA barcodes. Proc. R. Soc. B. 270: 313-322.

Hebert, P.D.N., E. H. Penton, J. M. Burns, D. H. Janzen, and W. Hallwachs. 2004. Ten species in one: DNA barcoding reveals cryptic species in the neotropical skipper butterfly Astraptes fulgerator. Proc. Natl. Acad. Sci. U.S.A. 101: 14812-14817.

Jenkins, C., T. A. Chapman, J. L. Micallef, and O. L. Reynolds. 2012. Molecular techniques for the detection and differentiation of host and parasitoid species and the implications for fruit fly management. Insects 3: 763-788.

Jinbo, U., T. Kato, and M. Ito. 2011. Current progress in DNA barcoding and future implications for entomology. Entomol. Sci. 14: 107-124.

Karim, S. 2000. Management of Helicoverpa armigera: a review and prospectus for Pakistan. Pakistan J. Biol. Sci. 3: 1213-1222.

Kimura, M. 1980. A simple method for estimating evolutionary rates of base substitutions through comparative studies of nucleotide sequences. J. Mol. Evol. 16: 111-120.

Krishnamurthy, P. K., and R. A. Francis. 2012. A critical review on the utility of DNA barcoding in biodiversity conservation. Biodivers. Conserv. 21: 1901-1919.

Lammers, J. W., and A. Macleod. 2007. Report of a pest risk analysis: Helicoverpa armigera (Hübner, 1808). (http:// www.fera.defra.gov.uk/plants/plantHealth/pestsDiseases/ documents/helicoverpa.pdf).

Laster, M. L., and D. D. Hardee. 1995. Intermating compatibility between North-American Helicoverpa zea and Heliothis armigera (Lepidoptera: Noctuidae) from Russia. J. Econ. Entomol. 88: 77-80.

Laster, M. L., and C. F. Sheng. 1995. Search for hybrid sterility for Helicoverpa zea in crosses between the North American Heliothis zea and Helicoverpa armigera (Lepidoptera: Noctuidae) from China. J. Econ. Entomol. 88: $1288-1291$.

Laster, M. L., C. E. Goodpasture, E. G. King, and P. Twine. 1985. Results from crossing the bollworms Helicoverpa armigera $\times$ H. zea in search of backcross sterility, pp. 146-147. In Proceedings, 1985, Beltwide Cotton Production and Research Conference National Cotton Council of America, Memphis, TN.

Li, Q. Q., D. Y. Li, H. Ye, X. F. Liu, W. Shi, N. Cao, and Y. Q. Duan. 2011. Using COI gene sequence to barcode two morphologically alike species: the cotton bollworm and the oriental tobacco budworm (Lepidoptera: Noctuidae). Mol. Biol. Rep. 38: 5107-5113.

Librado, P., and J. Rozas. 2009. DnaSP v5: A software for comprehensive analysis of DNA polymorphism data Bioinformatics 25: 1451-1452. (http://bioinformatics. oxfordjournals.org/content/25/11/1451).

Liu, H., and A. T. Beckenbach. 1992. Evolution of the Mitochondrial Cytochrome Oxidase II Gene among 10 Orders of Insects. Mol. Phylogenet. Evol. 1: 41-52.

Lyra, M. L., L. B. Klaczko, and A.M.L. Azeredo-Espin. 2009. Complex pattern of genetic distribution in populations of the New World Screwworm fly revealed by mitochondrial DNA markers. Med. Vet. Entomol. 23: 32-42.

Mallet, J., A. Korman, D. Heckel, and P. King. 1993. Biochemical genetics of Heliothis and Helicoverpa (Lepidop- 
tera: Noctuidae) and evidence for a founder event in Helicoverpa zea. Ann. Entomol. Soc. Am. 86: 189-197.

Nei, M. 1987. Molecular evolutionary genetics. Columbia University Press, New York, NY.

Nibouche, S., R. Bues, J. F. Toubon, and S. Poitout. 1998. Allozymepolymorphisms in the cotton bollworm Helicoverpa armigera (Lepidoptera: Noctuidae): comparison of African and European populations. Heredity 80: 438 - 445.

Nylander, J.A.A. 2004. MrAIC.pl. Program distributed by the author. Evolutionary Biology Centre, Uppsala University. (https://github.com/nylander/MrAIC).

Passoa, S. 2004. Identification guide to larval Heliothinae (Lepidoptera: Noctuidae) of quarantine significance. U.S. Dep. Agric. Report Columbus, OHIO 43212.

Pedgley, D. E. 1985. Windborne migration of Heliothis armigera (Hübner) (Lepidoptera: Noctuidae) to the British Isles. Entomol. Gazette. 36: 15-20.

Pogue, M. G. 2004. A new synonym of Helicoverpazea (Boddie) and differentiation of adult males of $H$. zea and $H$. armigera (Hübner) (Lepidoptera: Noctuidae: Heliothinae). Ann. Entomol. Soc. Am. 97: 1222-1226.

Reynolds, J., B. S. Weir, and C. C. Cockerham. 1983. Estimation for the coancestry coefficient: basis for a shortterm genetic distance. Genetics 105: 767-779.

Ronquist, F., and J. P. Huelsenbeck. 2003. MrBayes 3: Bayesian phylogenetic inference under mixed models. Bioinformatics 19: 1572-1574.

Rubinoff, D., S. Cameron, and K. Will. 2006. A genomic perspective on the shortcomings of mitochondrial DNA for "barcoding" identification. J. Heredity 97: 581-594.

Saitou, N., and M. Nei. 1987. The neighbor-joining method: a new method for reconstructing phylogenetic trees. Mol. Biol. Evol. 4: 406-425.

Sheng, C. F. 1993. Outbreak of Heliothis armigera in North China: possible causes and control strategies, pp. 841-844. In Proceedings, Beltwide Cotton Production Research Conferences. National Cotton Council of America, Memphis, TN.

Silva-Brandão, K. L., M. L. Lyra, and A.V.L. Freitas. 2009. Barcoding Lepidoptera: current situation and perspectives on the usefulness of a contentious technique. Neotrop. Entomol. 38: 441-451.

Slatkin, M. 1995. A measure of population subdivision based on microsatellite allele frequencies. Genetics 139: 457462.

Sievers, F., A. Wilm, D. G. Dineen, T. J. Gibson, K. Karplus, W. Li, R. Lopez, H. McWilliam, M. Remmert, J. Soding, et al. 2011. Fast, scalable generation of high-quality protein multiple sequence alignments using Clustal Omega. Mol. Syst. Biol. 7: 1-6.
Srivastava, C. P., R. Ahmad, R. Ujagir, and S. B. Das. 2005 Helicoverpa armigera management in pulses-present scenario and future strategies, pp: 265-286. In Recent Advances in Helicoverpa armigera Management. Indian Society of Pulses Research and Development, Kanpur, India.

Tamura, K., D. Peterson, N. Peterson, G. Stecher, M. Nei, and S. Kumar. 2011. MEGA5: molecular evolutionary genetics analysis using maximum likelihood, evolutionary distance, and maximum parsimony methods. Mol. Biol. Evol. 28: 2731-2739.

Tay, W. T., M. F. Soria, T. Walsh, D. Thomazoni, P. Silvie, G. T. Behere, C. Anderson, and S. Downes. 2013. A brave new world for an old world pest: Helicoverpa armigera (Lepidoptera: Noctuidae) in Brazil. PLoS ONE 8: e80134 2013

Venette, R. C., E. E. Davis, Z. Zaspel, H. Heisler, and M. Larson. 2003. Mini risk assessment, Old World bollworm, Helicoverpa armigera (Hubner) (Lepidoptera: Noctuidae). Cooperative Agricultural Pest Survey, Animal and Plant Health Inspection Service, US Department of Agriculture. (http:/ / www.aphis.usda.gov/plant health/ plant_pest_info/pest_detection/downloads/pra/ harmigerapra.pdf).

Wahlberg, N., and C. W. Wheat. 2008. Genomic outposts serve the phylogenomic pioneers: Designing novel nuclear markers for genomic DNA extractions of lepidoptera. Syst. Biol. 57: 231-242.

Wang, N. C., and Z. H. Li. 1984. Studies on the biology of cotton bollworm (Heliothis armigera Hübner) and tobacco budworm (Heliothis assulta Quenee). J. Shandong Agric. Univ. 1: 13-25.

Waugh, J. 2007. DNA barcoding in animal species: progress, potential and pitfalls. Bioessays 29: 188-197.

Whinnett, A., M. Zimmermann, K. R. Willmott, N. Herrera, R. Mallarino, F. Simpson, M. Joron, G. Lamas, and J. Mallet. 2005. Striking variable divergence times inferred across an Amazonian butterfly 'sature zone'. Proc. R. Soc. B 272: 2525-2533.

Widmer, M. W., and P. Schofield. 1983. Heliothis Dispersal and Migration. Tropical Development and Research Institute, London, United Kingdom.

Zhou, X., O. Faktor, S. W. Applebaum, and M. Coll. 2000. Population structure of the pestiferous moth Helicoverpa armigera in the eastern Mediterranean using RAPD analysis. Heredity 3: 251-256.

Received 17 September 2013; accepted 10 March 2014. 